AN. MED INTERNA (Madrid) Vol. 19, N. ${ }^{\circ}$ 1, pp. 23-26, 2002

\title{
Peritonitis espontánea por Campylobacter spp en pacientes con cirrosis hepática. Presentación de dos casos y revisión de la literatura
}

\author{
L. CALONGE RAVENTOS, O. LEN ABAD, R. BARTOLOMÉ*, T. FERNÁNDEZ \\ DE SEVILLA \\ Servicios de Medicina Interna y *Microbiología. Hospital General Universitario Vall \\ d'Hebrón. Barcelona.
}

\begin{abstract}
SPONTANEOUS PERITONITIS DUE TO CAMPYLOBACTER SPP IN PATIENTS WITH HEPATIC CIRRHOSIS. PRESENTATION OF TWO CASES AND REVIEW OF THE LITERATURE
\end{abstract}

\section{RESUMEN}

Los microorganismos del género Campylobacter son infrecuentes como agente etiológico de la peritonitis bacteriana espontánea (PBE). Presentamos 2 casos de PBE por Campylobacter jejuni diagnosticados en nuestro hospital junto a una revisión de la literatura mediante el sistema MEDLINE. En total encontramos 13 casos de PBE en los que se aisló Campylobacter sp en el líquido ascítico. Las principales características de estos casos fueron: la existencia de una cirrosis hepática evolucionada de etiología enólica, una alta positividad de los hemocultivos así como un índice de recidiva y una letalidad elevada.

PALABRAS CLAVE: Peritonitis bacteriana espontánea. Campylobacter spp.

\begin{abstract}
Campylobacter spp is not usual as the aetiology agent for spontaneous bacterial peritonitis. We report two cases of spontaneous peritonitis due to Campylobacter jejuni diagnosed in our hospital and review the literature trough MEDLINE data base. We found 13 cases of spontaneous peritonitis in which Campylobacter $s p$ was isolated from ascitic fluid. The main characteristics of these cases were: the existence of an evolved alcoholic cirrhosis, a high positivity of blood cultures as well as a high relapse index and letality.
\end{abstract}

KEY WORDS: Spontaneus bacterial peritonitis. Campylobacter spp.

Calonge Raventos L, Len Abad O, Bartolomé R, Fernández de Sevilla T. Peritonitis espontánea por Campylobacter spp en pacientes con cirro sis hepática. Presentación de dos casos y revisión de la literatura. An Med Interna (Madrid) 2002; 19: 23-26.

\section{INTRODUCCIÓN}

De los pacientes que presentan una descompensación ascítica de su cirrosis hepática, del 8 al $25 \%$ presentan un episodio de peritonitis bacteriana espontánea (PBE) en el momento del ingreso o lo desarrollan a lo largo del mismo. La mayoría de ellos están causados por bacilos gram negativos de procedencia intestinal, especialmente Escherichia coli (1). Las bacterias anaerobias o microaerófilas como Campylobacter spp se han implicado raramente en la PBE del cirrótico.

El género Campylobacter produce habitualmente infección entérica que se manifiesta como diarrea, dolor abdominal, fiebre y vómitos. La bacteriemia es una manifestación inusual que afecta preferentemente a pacientes inmunocomprometidos (2-10). A pesar de describirse en la literatura con mayor frecuencia las bacteriemias por Campylobacter spp en la última década $(3,11,12)$ gracias a la mejoría de las técnicas de cultivo (4) y el mayor número de pacientes con alteraciones del sistema inmunológico, especialmente los infectados por el virus de la inmunodeficiencia humana $(4-6,9,10,13)$, continúa siendo excepcional aislar a Campylobacter spp como agente causal de PBE. Por ello en el presente trabajo aportamos 2 pacientes con PBE por $C$. jejuni y realizamos una revisión de la literatura de aquellos casos en que también se ha conseguido aislar Campylobacter spp en el líquido ascítico en pacientes con PBE.

\section{CASO APORTADO}

Caso 1: Varón de 62 años, fumador de 30 cigarrillos/día y hábito enólico de más de $100 \mathrm{gr} / \mathrm{dia}$. Antecedentes patológicos de HTA, carcinoma escamoso de seno piriforme intervenido 4 años antes y actualmente en remisión. Cirrosis enólica Child $\mathrm{C}$ de 15 años de evolución con un ingreso previo por descompensación ascítica, que acudió al Hospital por distensión abdominal y fiebre. En la exploración física destacaba la presencia de ictericia cutaneomucosa, estigmas de hepatopatía crónica y hepatomegalia de 4 centímetros, semiología de ascitis y edemas maleolares. La analítica objetivó: bilirrubina total $9 \mathrm{mg} / \mathrm{dl}$, bilirrubina directa 4,65 mg/dl, Na $130 \mathrm{mEq} / \mathrm{L}$, AST $19 \mathrm{UI} / \mathrm{L}$, ALT 19 UI/L, fosfatasas alcalinas $230 \mathrm{UI} / \mathrm{L}$, GGT $22 \mathrm{UI} / \mathrm{L}$, LDH $279 \mathrm{UI} / \mathrm{L}$,

Trabajo aceptado: 14 de Julio de 2000

Correspondencia: Laura Calonge i Raventós. Carrer del Carme, 15-17, 2-2. 08001 Barcelona. 
proteínas 4,7 g/dl, albúmina 2,57 g/dl, hemoglobina $6,9 \mathrm{~g} / \mathrm{dl}$, hematocrito $19,6 \%$, VCM 114.4 fL, HCM 40,3 pg, leucocitos 4600/mm3 con fórmula normal, plaquetas $50000 / \mathrm{mm} 3$, tiempo de protrombina $40 \%$, HbsAg y Anti-VHC negativos. En la analítica del liquido ascítico se evidenció: glucosa $170 \mathrm{mg} / \mathrm{dl}$, proteínas $0,8 \mathrm{~g} / \mathrm{dL}$, amilasa $27 \mathrm{UI} / \mathrm{l}$, ADA 10 UI/L, LDH 206 UI/L, 14400 células (45\% polimorfonucleares). Los hemocultivos y el cultivo de líquido ascítico resultaron positivos para Campylobacter jejuni, que en el antibiograma fue sensible a ampicilina, amoxicilina-clavulánico, gentamicina, eritromicina y doxiciclina y resistente a cefalotina, cotrimoxazol, ácido nalidíxico, norfloxacino y ciprofloxacino. Los coprocultivos fueron negativos. Se inició tratamiento con amoxicilina-clavulánico a dosis de 1 gr cada 8 horas por vía endovenosa durante 14 días con buena evolución clínica. La fiebre remitió a las 48 horas.

Caso 2: Varón de 54 años, fumador de 20 cigarrillos/día y hábito enólico de más de 100 grs/día hasta hacía 15 años. Antecedentes patológicos de cirrosis enólica Child $\mathrm{C}$ diagnosticada 5 años antes, en programa de transplante hepático, con varios ingresos por hemorragia digestiva alta, descompensaciones ascíticas y encefalopáticas, que consultó por aumento del perímetro abdominal y síndrome confusional. A la exploración física destacaban estigmas de hepatopatía crónica, ictericia conjuntival, asterixis y semiología de ascitis. En la analítica general se observó: glucosa $300 \mathrm{mg} / \mathrm{dL}$, bilirrubina total/directa 5,1/2,2 mg/dL, Na $123 \mathrm{mEq} / \mathrm{L}, \mathrm{K} 4,5 \mathrm{mEq} / \mathrm{L}$, AST $86 \mathrm{UI} / \mathrm{L}$, ALT $50 \mathrm{UI} / \mathrm{L}$, fosfatasas alcalinas $652 \mathrm{UI} / \mathrm{L}$, GGT $261 \mathrm{UI} / \mathrm{L}$, proteínas $6,3 \mathrm{~g} / \mathrm{dL}$, albúmina $3,15 \mathrm{gr} / \mathrm{dL}$, hemoglobina $8,6 \mathrm{~g} / \mathrm{dL}$, hematocrito $25 \%$, VCM $90 \mathrm{fl}$, plaquetas $85000 / \mathrm{mm} 3$, leucocitos 4600/mm3 con $80 \%$ polimorfonucleares, VSG $43 \mathrm{~mm}$, tiempo de Quick 50\%. En la analítica del líquido ascítico se evidenció: glucosa $255 \mathrm{mg} / \mathrm{dL}$, proteínas $0,5 \mathrm{~g} / \mathrm{dL}$, amilasa $10 \mathrm{UI} / \mathrm{L}, \mathrm{LDH}$ $50 \mathrm{UI} / \mathrm{L}, 250$ polimorfonucleares. Los hemocultivos y el cultivo del líquido ascítico resultaron positivos para C. jejuni, que en el antibiograma fue sensible a la eritromicina, doxicicliina, ampicilina, gentamicina y amoxicilina-clavulánico, y resistente a acido nalidíxico, cefalotina, ciprofloxacino, norfloxacino y trimetroprim. Se inició tratamiento con eritromicina endovenosa durante tres semanas con lo que permaneció afebril durante el ingreso. Requirió varias paracentesis evacuadoras. Un mes después de finalizar el tratamiento antibiótico ingresó de forma electiva para la realización de trasplante hepático. En el acto quirúrgico se evidenció líquido ascítico purulento que motivó la suspensión del transplante. La evolución posterior del paciente fue desfavorable a pesar del tratamiento antibiótico.

\section{DISCUSIÓN}

Los microorganismos del género Campylobacter son bacilos gram negativos microaerófilos, móviles (11). La vía de contagio en el ser humano es fundamentalmente a través de alimentos y aguas contaminadas (fecal-oral). Actualmente se reconocen 20 especies. Los que con mayor frecuencia causan enfermedad son: $C$. jejuni, $C$. fetus y $C$. coli. Pueden causar tanto enfermedad intestinal como extraintestinal (14). Cuando la infección se produce en pacientes inmunocompetentes se suele manifestar en forma de enterocolitis. Es el segundo microorganismo causal de gastroenteritis después del género Salmonella (3). Sin embargo, cuando la infección afecta a pacientes inmunodeprimidos puede desarrollarse un cuadro sistémico. Las enfermedades subyacentes que predisponen a enfermedad sistémica por Campylobacter spp son: cirrosis hepática, neoplasias, diabetes mellitus, infección por virus inmunodeficiencia humana, tratamiento inmunosupresor, cardiopatías, insuficiencia renal crónica, lupus eritematoso sistémico, hipogammaglobulinemias, tuberculosis pulmonar $\mathrm{y}$ desnutrición grave $(2-4,6-10,13,15,16)$.
Presentamos 2 casos de PBE en los que $C$. jejuni ha podido ser aislado en el líquido ascítico recogidos en nuestro hospital. Tras realizar una exhaustiva revisión de la literatura mediante el sistema MEDLINE sólo hemos encontrado 13 casos adicionales de PBE por el género Campylobacter (15-26). Únicamente hemos considerado los pacientes en los que se ha identificado Campylobacter spp en el líquido ascítico, sin tener en cuenta aquellos casos de bacteriemia con ascitis neutrocítica y cultivo negativo de líquido ascítico. En la tabla I reflejamos las principales características de los 13 casos recogidos en la literatura junto con los 2 nuestros.

La edad media de los pacientes fue de 60 años. Doce $(80 \%)$ de los 15 pacientes eran varones. En todos ellos la cirrosis hepática estaba en una fase muy evolucionada (grado Child C de la clasificación de Child-Pugh), y en todos los casos excepto en dos en los que la hepatopatía no estaba filiada, la etiología era alcoholica. Exclusivamente en un paciente se detectaron anticuerpos frente el virus de la hepatitis C. Otro enfermo estaba afecto de un hepatocarcinoma injertado sobre la cirrosis.

Nueve de las PBE fueron debidas a C. Jejuni (60\%), en 3 se aisló $C$. fetus y en uno $C$. coli. Cuatro (27\%) de los pacientes presentaron un cuadro de diarrea autolimitado previo al ingreso o durante el mismo, pero sólo en 2 se aisló $C$. jejuni en heces.

La presentación clínica más frecuente fue fiebre y/o dolor abdominal. La mayoría no presentaban signos de irritación peritoneal a la exploración abdominal. Queremos destacar que en 3 pacientes el contaje de polimorfonucleares en líquido ascítico mostró una cifra alrededor de 250 por lo que fue el cultivo el que confirmó el diagnóstico.

En un $66 \%$ de los pacientes los hemocultivos fueran positivos con lo que se podría sugerir que el origen de la PBE es por diseminación hematógena.

En el tratamiento fueron utilizados diferentes antibióticos: macrólidos, aminoglucósidos, cefalosporinas de $2^{\mathrm{a}}$ y $3^{\mathrm{a}}$ generación y amoxicilina+clavulánico, durante un período mínimo de 2 semanas. En general se acepta que la sensibilidad de Campylobacter sp es elevada para eritromicina $(5,12)$ y aminoglucósidos $(4,11,12)$ aunque ya se han descrito cepas resistentes (12). La amoxicilina-clavulánico también se ha demostrado como una alternativa eficaz (3). Es destacable la progresiva resistencia a las fluorquinolonas $(12,13)$. Si bien en la literatura revisada se recomienda realizar tratamiento durante 3 a 4 semanas por el carácter recidivante de estas infecciones $(11,15,16,19)$, hemos observado que todos los pacientes que fueron tratados únicamente durante 12 o 14 días presentaron una buena evolución.

Fallecieron 6 pacientes (40\%) si bien creemos que la mortalidad estuvo más relacionada con la severidad de la cirrosis hepática. De los 6 casos, 2 fallecieron posteriormente a la curación de la infección del líquido ascítico.

No hemos encontrado diferencias en cuanto a presentación, evolución y respuesta al tratamiento en las PBE causadas por las diferentes especies de Campylobacter.

En definitiva, podemos concluir que la peritonitis espontánea por Campylobacter spp en pacientes con cirrosis es un acontecimiento extremadamente inusual. Los casos reportados hasta la actualidad pertenecen a pacientes con cirrosis hepática de origen enólico en su gran mayoría (80\% en la revisión y 20\% sin etiología filiada). El comportamiento clínico y la respuesta al tratamiento es similar a la causada por los bacilos gramnegativos 
TABLA I

\section{CASOS PUBLICADOS DE PERITO NITIS ESPONTÁNEA POR CAM PYLOBACTER SPP}

\begin{tabular}{|c|c|c|c|c|c|c|c|}
\hline Sexo/Edad & $\begin{array}{l}\text { Enfermedad } \\
\text { hepática }\end{array}$ & $\begin{array}{l}\text { PMN en } \\
\text { líquido } \\
\text { ascítico }\end{array}$ & $\begin{array}{l}\text { Cultivo } \\
\text { Líquido } \\
\text { ascítico }\end{array}$ & Hemocultivo & Coprocultivo & Evolución & Referencia \\
\hline $\begin{array}{l}\text { Varón } \\
56\end{array}$ & $\begin{array}{l}\text { Cirrosis alcohólica } \\
\text { Hepatocarcinoma }\end{array}$ & 5760 & C. jejuni & C. jejuni & C. jejuni & Exitus & 24 \\
\hline $\begin{array}{l}\text { Varón } \\
56\end{array}$ & $\begin{array}{l}\text { Cirrosis } \\
\text { Alcohólica }\end{array}$ & 11040 & C. jejuni & C. jejuni & No realizado & Exitus & 23 \\
\hline $\begin{array}{l}\text { Varón } \\
76\end{array}$ & $\begin{array}{l}\text { Cirrosis } \\
\text { Alcohólica }\end{array}$ & 807 & C. jejuni & Negativo & C. jejuni & Favorable & 16 \\
\hline $\begin{array}{l}\text { Varón } \\
64\end{array}$ & Cirrosis & 368 & C. fetus & Negativo & No realizado & Favorable & 19 \\
\hline $\begin{array}{l}\text { Varón } \\
59\end{array}$ & $\begin{array}{l}\text { Cirrosis } \\
\text { Alcohólica }\end{array}$ & $\begin{array}{c}\text { no } \\
\text { disponibles }\end{array}$ & C. fetus & Negativo & No realizado & Exitus & 20 \\
\hline $\begin{array}{l}\text { M ujer } \\
36\end{array}$ & $\begin{array}{l}\text { Cirrosis } \\
\text { Alcohólica } \\
\text { VHC positivo }\end{array}$ & 1792 & C. fetus & C. fetus & Negativo & Favorable & 17 \\
\hline $\begin{array}{l}\text { Varón } \\
46\end{array}$ & $\begin{array}{l}\text { Cirrosis } \\
\text { Alcohólica }\end{array}$ & 2565 & C. coli & C. coli & No realizado & Exitus & 18 \\
\hline $\begin{array}{l}\text { M ujer } \\
72\end{array}$ & $\begin{array}{l}\text { Hepatopatía } \\
\text { No filiada }\end{array}$ & 600 & C. jejuni & Negativo & Negativo & Favorable & 15 \\
\hline $\begin{array}{l}\text { Varón } \\
60\end{array}$ & $\begin{array}{l}\text { Cirrosis } \\
\text { Alcohólica }\end{array}$ & 1370 & C. jejuni & C. jejuni & No realizado & Exitus & 25 \\
\hline $\begin{array}{l}\text { Varón } \\
48\end{array}$ & $\begin{array}{l}\text { Cirrosis } \\
\text { Alcohólica }\end{array}$ & 300 & C. jejuni & No realizado & No realizado & Favorable & 26 \\
\hline $\begin{array}{l}\text { Varón } \\
70\end{array}$ & Cirrosis & 280 & C. fetus & C. fetus & Negativo & Favorable & 21 \\
\hline $\begin{array}{l}\text { Varón } \\
62\end{array}$ & $\begin{array}{l}\text { Cirrosis } \\
\text { Alcohólica }\end{array}$ & 260 & C. jejuni & C. jejuni & Negativo & Favorable & 22 \\
\hline $\begin{array}{l}\text { Mujer } \\
81\end{array}$ & $\begin{array}{l}\text { Cirrosis } \\
\text { Alcohólica }\end{array}$ & 14760 & C. fetus & C. fetus & Negativo & Favorable & 22 \\
\hline $\begin{array}{l}\text { Varón } \\
62\end{array}$ & $\begin{array}{l}\text { Cirrosis } \\
\text { Alcohólica }\end{array}$ & 6480 & C. jejuni & C. jejuni & Negativo & Favorable & Caso propio \\
\hline $\begin{array}{l}\text { Varón } \\
54\end{array}$ & $\begin{array}{l}\text { Cirrosis } \\
\text { Alcohólica }\end{array}$ & 250 & C. jejuni & C. jejuni & No realizado & Exitus & Caso propio \\
\hline
\end{tabular}

PM N: cifra de leucocitos polimorfonucleares $/ \mathrm{mm}^{3}$. VHC: virus de la hepatitis $\mathrm{C}$.

más habituales en esta infección como Escherichia coli. Aunque la mortalidad observada es del $40 \%$ creemos que probablemente está más en relación con la severidad de la enfermedad hepática subyacente que con la patogenicidad del microorganismo.

\section{AGRADECIMIENTOS}

A la Dra. Ana Maria Planes y al Dr. Vicenç Falcó por sus consejos en la realización de este trabajo. 


\section{Bibliografía}

1. Such J, Soriano G, Guarner C. Peritonitis bacteriana espontanea. Rev Esp Enf Dig 1992; 81: 341-7.

2. Vargas J, Lozano MC, Andreu J, Martín E. Bacteriemia por Campylobacer jejuni en paciente cirrótico con ascitis. Med Clin (Barcelona) 1992; 99: 274.

3. Pigrau C, Bartolomé R, Almirante B, Planes AM, Gavalda J, Pahissa A. Bacteremia due to Campylobacter species: clinical findings and antimicrobial susceptibility patterns. Clin Infect Dis 1997; 25: 1414-20.

4. Tee W, Mijch A. Campylobacter jejuni bateremia in human inmunodeficiency virus (HIV) infected and non-HIV infected patients: comparison of clinical features and review. Clin Infect Dis 1998; 26: 91-6.

5. Sanchez A, Iñigo MA, Gonzalez M, Torres M. Bacteremia por Campylobacter jejuni en pacientes con síndrome de inmunodeficiencia adquiri da y cirrosis hepática. Med Clin (Barcelona) 1992; 99: 438-9.

6. Lucena F, Lopez J, Monetero E, Saiz E, Cuadros JA. Bacteriemia en pacientes HIV positivos y cirrosis hepática por Campylobacter fetus spp. Enf Infec Microbiol Clín 1990; 8: 329-330.

7. Casas JM, Perez I, Jaime ML, Merino J. Bacteremia espontánea por Campylobacter jejuni. Rev Clin Esp 1990; 186: 246.

8. Santamaria S, Saez F, Marna C, Lopez A. Bacteremia por Campylobacter jejuni y vasculitis leucocitoclástica en un paciente cirrótico. Enf Infec Microbiol Clín 1993; 11: 229.

9. Pigrau C, Almirante B, Bartolomé R, Pahissa A. Bacteriemia por Campylobacter spp en pacientes con infección por VIH. Med Clin (Barcelona) 1993; 103: 239.

10. Chaves F, Dronda F, Gonzalez A. Bacteriemia por Campylobacter jejuni en pacientes infectados por VIH. Med Clin (Barcelona) 1993; 100: 514.

11. Pedro-Botet ML, Sabrià M, Carrasco I, Arnal J, Comdon MJ. Bacteremia por Campylobacter fetus: Interés e incidencia crecientes. Med Clin Barcelona) 1991; 97: 262-264.

12. Font C, Cruceta A, Moreno A, Miró O, Coll-Vinent B, Almela M, Mensa J. Estudio de 30 pacientes con bacteriemia por Campylobacter spp. Med Clin (Barcelona) 1997; 108: 336-340.

13. Knobel H, Lopez Colomes JL, Saballs P, Drobnic L. Formas inusuales de bacteremia por Campylobacter jejuni en pacientes con infección por el VIH. Med Clin (Barcelona) 1994; 103: 23.
14. Allos BM, Blaser MJ. Campylobacter jejuni and the expanding spectrum of related infections. Clin Infect Dis 1995; 20: 1092-110.

15. Roca B, Elbar FA, Sevadilla L, Arenas M. Peritonits bacteriana espontánea causada por Campylobacter jejuni. Rev Clin Esp 1993; 193: 214-5.

16. Domingo P, Mirelis B, Gimeno A, Cabezas R. Peritonitis espontánea por Campylobacter jejuni en un paciente cirrótico. Med Clin (Barcelona) $1985 ; 84: 416$.

17. Saro C, Garcia R, Suarez A, Roman F, Rodriguez A, Navascues LA. Campylobacter fetus: un germen infrecuente causante de peritonitis bacteriana espontánea del cirrótico. Rev Esp Enf Dig 1994; 86: 845-7.

18. Hoi H, Zuckerman MJ, Polly SM. Spontaneus bacterial peritonitis due to Campylobacter coli. Gastroenterol 1987; 92: 2024-5.

19. Rodriguez Ramos C, Galán F, García Tapia AM, Porcel A, Amaya A, Martín L. Peritonitis bacteriana espontánea por Campylobacter fetus. Rev Esp Enf Dig 1997; 89: 481-3.

20. Targan SR, Chow AV, Guze LB. Spontaneous bacterial peritonitis due to Campylobacter fetus. Gastroenterol 1976; 71: 311-3.

21. Texier J, Couzigou P, Mormede M, Fourche J, Beraud C. Isolement d'un Campylobacter fetus spp d'un liquide d'ascite iors d'une septicemie chez un cirrhotique. Med Mal Infect 1985; 4: 173-4.

22. Chassagne P, Borreda D, Bonnet B, Manchon ND, Pariente AE, Bercoff E. Infections du liquide d'ascite a Campylobacter: 2 observations: Gastroenterol Clin Biol 1991;15: 96-97.

23. Mc Neil NI, Butto S, Ridgway GL. Spontaneus bacterial peritonitis due to Campylobacter jejuni. Postgrad Med J 1984; 60: 487-8.

24. Vermeij CG, Van Dissel JT, Veeenendaal RA, Lawers LB, Van Holk B. Campylobacter jejuni peritonitis in a patient with liver cirrhosis. Eur $\mathbf{J}$ Gastroenterol Hepatol 1996; 8: 1219-21.

25. Granje JD, Amiat X, Granje V, Gutman L, Biour M, Bodin F. Amoxicillin-Clavulanic acid therapy of spontaneus bacterial peritonitis. A prospective study of twenty-seven cases in cirrhotic patients. Hepatology 1990; 11: 360-364.

26. Castiella A, Yuste R, Gonzalez F, Martinez S, Alzate L, Arriola JA Infection du liquide d'ascite a Campylobacter jejuni chez un malade atteint de cirrhose. Une nouvelle observation avec une presentation atypique. Gastroenterol Clin Biol 1994; 18: 797-798. 